\title{
L'histoire de Méléagre vue par Ovide ou de quoi le tison des Parques est-il l'emblème?
}

\section{Jacqueline Fabre-Serris}

\section{(2) OpenEdition \\ 1 Journals}

Édition électronique

URL : http://journals.openedition.org/edl/195

DOI : 10.4000/edl. 195

ISSN : 2296-5084

Éditeur

Université de Lausanne

\section{Édition imprimée}

Date de publication : 15 décembre 2011

Pagination : 149-166

ISBN : 978-2-940331-26-0

ISSN : 0014-2026

\section{Référence électronique}

Jacqueline Fabre-Serris, «L'histoire de Méléagre vue par Ovide ou de quoi le tison des Parques est-il l'emblème? », Études de lettres [En ligne], 3-4 | 2011, mis en ligne le 15 décembre 2014, consulté le 19 décembre 2020. URL : http://journals.openedition.org/edl/195; DOI : https://doi.org/10.4000/edl.195 


\section{L'HISTOIRE DE MÉLÉAGRE VUE PAR OVIDE OU DE QUOI LE TISON DES PARQUES EST-IL L'EMBLÈME?}

S'inspirant de la technique narrative des mythographes, Ovide raconte l'histoire de Méléagre d'une façon linéaire, sous la forme de séquences conçues et enchaînées de façon à donner une cohérence à l'existence de ce héros épique. La construction narrative met en évidence l'importance du rôle joué dans la vie de Méléagre par les femmes: Diane, Atalante, sa mère, ses sœurs. Le tison magique auquel sa vie est attachée est l'image de cette domination dont Méléagre n'a pas conscience. Donné par les Parques à sa mère, à qui elles délèguent leur droit d'impartir une durée à la vie humaine, il est l'emblème de ces liens invisibles et tout-puissants que chaque homme a, de sa naissance à sa mort, avec les femmes de sa famille, les unes et les autres étant plus ou moins interchangeables.

Comment raconter les mythes? La question se posait déjà dans l'Antiquité, où la tradition était beaucoup plus riche et diverse que celle dont nous disposons. Aujourd'hui, nous avons, à côté des textes antiques, les notices des dictionnaires mythologiques qui sont conçues sur le modèle narratif des textes mythographiques antiques et dont une des principales sources est constituée par les récits des Métamorphoses d'Ovide. Une convergence qui n'a rien de surprenant, Ovide s'étant inspiré de la pratique narrative des mythographes, et ses récits ayant à leur tour inspiré certains d'entre eux.

Ce que l'auteur des Métamorphoses a emprunté aux mythographes et qui a fait la radicale nouveauté de son texte, c'est un choix narratif: le fait de raconter l'histoire d'un personnage d'une façon linéaire, à partir de sa naissance - ou d'un épisode marquant de sa vie - jusqu'à sa mort, sous la forme de séquences constituées de résumés d'événements ou de scènes mettant en valeur un moment fort. L'analyse de cette technique 
servira de fil conducteur à mon étude d'une histoire qui fait doublement écho à la thématique de ce livre, puisqu'elle inclut un objet magique ${ }^{1}$ et qu'Ovide a racontée de façon à mettre en évidence le rôle des femmes dans l'existence du héros auquel l'objet est associé. Je veux parler de l'histoire de Méléagre et du tison magique auquel sa vie est attachée par les Parques dès sa naissance et que sa mère, Althée, garde caché pour protéger son fils jusqu'au moment où elle y verra un moyen de venger ses frères.

La tradition littéraire relative à Méléagre est exceptionnellement riche, tant du point de vue des genres - épique, lyrique, tragique, comique, historique, mythographique... - que des périodes: archaïque, classique, hellénistique pour la Grèce, républicaine et impériale pour Rome ${ }^{2}$. Autant dire qu'Ovide se trouvait face à plusieurs versions. Je voudrais montrer qu'il a choisi de raconter l'histoire de Méléagre sous la forme d'épisodes successifs sélectionnés dans diverses versions antérieures avec le projet de donner un sens à l'ensemble narratif ainsi constitué, en proposant de l'enchaînement de ces épisodes une interprétation qui transforme le cours de l'existence du héros en destin.

C'est une thèse expliquant l'origine des actes et de la mort de Méléagre qui se dessine, en effet, peu à peu sous les yeux du lecteur, s'il se met dans les pas de l'auteur qui l'engage, par sa construction narrative, à effectuer cette démarche et s'il relie tous les fils de la trame de la vie qui lui est racontée. Méléagre appartenant à la catégorie des chefs

I. La présence d'un objet magique est rare dans les mythes, mais cela ne rapproche pas pour autant l'histoire de Méléagre d'un conte, du moins dans l'état actuel de nos connaissances sur ce genre narratif dans l'Antiquité. Le cas des Métamorphoses d'Apulée, présenté par son auteur comme un conte milésien, est à cet égard significatif. S'il ne nous est pas possible d'évaluer ce que le texte doit au sermone Milesio (1.1), la traduction que Cornelius Sisenna fit des Fables milésiennes d'Aristide de Milet ne nous étant pas parvenue, il est toutefois probable - je renvoie ici aux analyses de G. B. Conte (Profilo storico della letteratura latina, p. 268) - que la place donnée par Apulée à la magie relève davantage d'un choix d'auteur que d'une contrainte du genre. Pour G. B. Conte en effet, Apulée a donné à l'élément magique une importance sans commune mesure avec ce qui devait être le cas dans le texte grec qui lui a servi de modèle, la magie intervenant en particulier dans les premiers épisodes, qui correspondent à la volonté de l'auteur de mettre en évidence la nouveauté de son ouvre par rapport au genre dans lequel elle $s$ 'insère.

2. Pour une analyse des différentes versions du mythe de Méléagre et une discussion sur les hypothèses développées par les critiques, voir P. Grossardt, Die Erzählung von Meleagros. 
d'expéditions héroïques, ce n'est pas un des moindres paradoxes de la reconfiguration ovidienne de ce mythe grec que de suggérer au lecteur romain de réfléchir à la fois sur le point de vue des femmes et sur le type de rapports que les hommes ont, sans le savoir, avec celles de leur famille.

La transition, qu'Ovide a choisie d'insérer entre le récit de la métamorphose de Perdix à Athènes et la longue narration qui se termine par celle des sœurs de Méléagre, mentionne deux noms, celui de Méléagre et celui de Diane, un rapprochement significatif comme on le verra. La renommée du roi d'Athènes, Thésée, s'étant répandue en Argolide et en Achaïe, Calydon, écrit Ovide, quamuis Meleagron haberet (v. 270) ${ }^{3}$, l'appela pour combattre un sanglier, famulus uindexque Dianae (v. 272) ${ }^{4}$. Calydon appelle Thésée, mais c'est dans la vie de Méléagre que Diane va intervenir parce que le chef de la communauté à laquelle il appartient, et qui est aussi son père, ne lui a pas rendu l'hommage qu'il lui devait. Les récoltes ont été abondantes. Diane est la seule divinité qu'EEnée a oublié d'honorer. La colère la saisit, une réaction ainsi commentée: Tangit et ira deos (v. 379) 5 . Dans l'Enéide, Virgile s'interrogeait: [...] tantae animis caelestibus irae? (1.11) ${ }^{6}$. Ovide donne ici la parole à une déesse, qui déclare qu'elle se vengera en deux vers ponctués d'emportements passionnels: at non impune feremus, / quaeque inhonoratae, non et dicemur inultae (v. 279-280) ${ }^{7}$, qui à tout le moins manquent de la dignité et de la mesure attendues chez un dieu. On trouve quasiment la même formulation de départ, au livre 5 des Fastes, dans la bouche d'une autre déesse négligée, Flora, qui théorise la raison de la bienveillance et du courroux divin, en prenant précisément... l'exemple de Méléagre:

Nos quoque tangit honor: festis gaudemus et aris turbaque caelestes ambitiosa sumus [...]

At si neglegimur, magnis iniuria poenis soluitur et iustum praeterit ira modum

3. "quoiqu’elle eût Méléagre.»

4. "serviteur et vengeur de Diane»

5. «La colère touche aussi les dieux.» Les traductions d'Ovide présentées dans cet article sont celles de l'auteur.

6. "[...] y a-t-il d'aussi grandes colères dans l'âme des dieux du ciel?». Les traductions de Virgile présentées dans cet article sont celles de l'auteur.

7. «Mais ce n'est pas impunément, et je ne le supporterai pas, que je serai laissée sans honneur, et on ne dira pas non plus que je ne me suis pas vengée.» 
Respice Thestiaden: flammis absentibus arsit;

causa est quod Phoebes ara sine igne fuit (v. 297-298, 303-306) ${ }^{8}$.

Le dernier vers est typique de l'ingenium d'Ovide et de sa façon d'interpréter un mythe en reliant certaines de ses séquences. Dans ce passage des Fastes, le poète identifie dans l'oubli d'honneurs dus à Diane et dans la colère qui saisit alors la déesse la cause de la mort du fils du coupable, en notant l'existence d'un élément commun à deux séquences de l'histoire: des flammes absentes, mais qui dans le second cas, qui est le pendant punitif du premier, brûlent à distance, conformément à l'idée que la nature d'un châtiment est en relation avec la faute commise. La lecture développée dans les Métamorphoses est une espèce d'amplification de ce brillant point de vue, mais pas seulement: dans la mesure où elle prend en compte tous les protagonistes féminins et masculins de l'histoire et inclut, de ce fait, un épisode postérieur à la mort du héros.

Rappelons en quelques mots le premier épisode. Diane envoie un sanglier gigantesque qui ravage les récoltes pour lesquelles elle aurait dû être honorée. Le fils du fautif, Méléagre, monte une expédition avec l'élite des jeunes gens de son temps qui appartiennent à la génération des Argonautes. Aucun détail du récit ne laisse supposer que la troupe sait à quoi s'en tenir sur l'implication de la déesse, jusqu'aux vers 394-395, sur lesquels je reviendrai. Le catalogue des héros qui prirent part à cette chasse extraordinaire se termine par l'évocation de la seule femme de l'expédition, qui n'est pas nommée, mais désignée par une périphrase soulignant sa valeur: nemorisque decus Tegeaea Lycaei (v. 317) ${ }^{9}$. Notons que, dans l'Enéide, le mot decus est utilisé, en tant qu'épithète, exclusivement à propos de femmes: Iris, en 9.18 (decus caeli), Séléné en 9.405 (astrorum decus), Camille en 11.508 (decus Italiae uirgo) ${ }^{10}$, Juturne en 12.142 (decus fluniorum).

8. "Nous aussi les honneurs nous touchent; nous nous réjouissons des fêtes et des autels et nous sommes dans le ciel une troupe avide d'honneurs [...] mais si nous sommes négligés, l'injure qui nous est faite est payée par de grands châtiments et notre colère dépasse la juste mesure. Vois le petit-fils de Thestius: il fut brûlé par des flammes absentes. La cause en est que l'autel de Phébé était sans feu.»

9. "la Tégéenne, gloire du bois lycéen».

Io. On peut ajouter l'emploi du mot à propos des jeunes guerrières de l'entourage de Camille: Larina, Tulla et Tarpeia, quas ipsa decus sibi dia Camilla / delegit pacisque 
La description de son cultus désigne clairement en Atalante un doublet de Diane, une façon de signaler d'entrée au lecteur que la déesse se manifestera par son intermédiaire. J'en commente rapidement les détails, qui, chez Ovide, sont canoniques dans les descriptions des vierges chasseresses, émules et compagnes de Diane, et qui évoquent donc aussi la déesse des forêts. La junctura fibula uestem (v. 318), qui introduit la description de l'agrafe tenant le vêtement d'Atalante renvoie au livre 2, où elle est employée à propos de l'une d'entre elles, Callisto (2.412), qualifiée de miles ... Phoebes (v. 415) ${ }^{11}$. L'expression utilisée pour décrire la coiffure d'Atalante - crinis [...] nodum collectus in unum (v. 319) reprend des termes employés à propos de Diane: au livre 3, une de ses compagnes, Isménis, qui la prépare pour son bain, capillos / colligit in nodum (v. 169-170) ${ }^{12}$.

Plus originale est la description du visage d'Atalante:

[...] facies, quam dicere uere uirgineam in puero, puerilem in uirgine possis (v. 322-323) ${ }^{13}$.

Cette description précédant la notation du coup de foudre de Méléagre à sa vue (v. 324-325), le lecteur est engagé à supposer un processus de cause à effet. Mais qu'est-ce qui est signalé ici? Une indécision sexuelle qui ferait le charme d'Atalante, du moins aux yeux du héros, poussé jusque-là uniquement, on l'a lu au vers 300 , par une cupidine laudis? Ou faut-il prendre aussi cette double réflexion, qui établit une équivalence entre un garçon avec un air de fille et une fille avec un visage de garçon, comme deux façons d'indiquer que l'intrusion d'un élément à l'aspect sexuel ambigu va perturber une activité considérée traditionnellement comme purement masculine?

La naissance de l'amour chez Méléagre est évoquée par la métaphore des flammes, qui ne sont pas visibles - [...] flammas latentes / hausit (v. 325-526) ${ }^{14}$ - ce qui serait banal chez tout autre que lui. Ovide

bonas bellique ministras (11.657-658); "que la divine Camille s'est choisi elle-même pour lui faire honneur et bien la servir dans la paix et dans la guerre.»

II. "soldat ... de Phébé».

I2. «rassemble ses cheveux en un nœud».

I3. " [...] un visage dont on aurait vraiment pu dire qu'il était celui d'une jeune fille chez un jeune garçon et d'un jeune garçon chez une jeune fille».

I4. "il fut envahi de flammes secrètes». 
marque ensuite par une parole le moment fort du rassemblement des chasseurs. Sous l'effet du sentiment qui l'envahit, Méléagre s'exclame:

«o felix, siquem dignabitur [...]

ista uirum» (v. 326) ${ }^{15}$.

Ces mots, calqués sur ceux d'Ulysse à Nausicaa, qu'ils inversent en donnant une position forte à la femme ${ }^{16}$, enclenchent la marche du destin: chaque geste du héros sera motivé par l'espoir d'être celui qu'Atalante choisira.

Le récit de la chasse confirme l'utilisation de la jeune fille comme instrument de vengeance. Les coups des hommes qui auraient pu porter sont rendus inefficaces par Diane. L'un d'entre eux, Jason, croit pouvoir réussir avec l'aide d'Apollon qu'il implore. Le dieu accède à ses prières qua potuit (v. 352) ${ }^{17}$. Aussi son protégé touche-t-il l'animal, mais sans le blesser parce que Diane enlève l'embout en fer du javelot ${ }^{18}$. Cette tentative plus réussie que les autres a un effet négatif: le sanglier, rendu furieux, se jette sur un groupe de jeunes gens, en blesse deux et en tue un troisième qui cherchait à fuir et se préparait à tourner le dos. Nestor agit peu glorieusement, lui aussi, mais il s'en tire: grimper à un arbre lui permet d'échapper à la mort. Les autres chasseurs hésitent ou échouent.

Le premier trait efficace est tiré par Atalante:

$$
\begin{gathered}
{[\ldots] \text { celerem Tegeaea sagittam }} \\
\text { imposuit neruo sinuatoque expulit arcus (v. 380-381) }{ }^{19} \text {. }
\end{gathered}
$$

Ce coup est apparemment dû à sa seule valeur puisquaucune mention n'est faite d'une quelconque intervention de la déesse. La blessure causée est légère mais incontestable: quelques gouttes de sang ont rougi

15. «Heureux l'homme qu'elle jugera digne d'elle!»

I6. Comme le note Ch. Segal ("Ovid's Meleager and the Greeks», p. 314), «his expression reverses the usual primacy of male choice in such matters. In the oldest and most famous model, for instance, the man has the initiative: to Odysseus, addressing Nausicaa, "most blessed [is] that man who loads you down with bride-gifts and brings you to his home” (Od., 6.158-159).»

I7. "autant que possible».

I8. [...] ferrum Diana uolanti / abstulerat iaculo, lignum sine acumine uenit (v. 353-354).

19. "[...] la Tégéenne a posé une flèche rapide sur la corde et l'a fait jaillir de son arc après l'avoir courbé». 
les poils de l'animal. Ovide note que Méléagre s'en réjouit plus encore qu'Atalante et qu'il est d'ailleurs le premier à avoir vu le sang perler. Cette séquence est elle aussi ponctuée par une parole tragique, dans la mesure où l'avis proclamé est loin d'être partagé: "meritum" dixisse "feres uirtutis honorem" (v. 387) ${ }^{20}$. Quoique brève, la proclamation de Méléagre contient trois mots-clefs: uirtus (la qualité propre aux hommes, revendiquée par eux et donc implicitement déniée aux femmes), honor (ce que tous les jeunes gens de l'expédition visent, chacun pour soi) et meritum (un adjectif appliqué à ce qui est sans contestation, mais qui sera précisément mis en doute).

Le mot de Méléagre est d'ailleurs immédiatement contredit par l'attitude de ses compagnons qui se comportent comme si le uirtutis honor était encore en débat:

erubuere uiri seque exhortantur et addunt cum clamore animos [...] (v. 388-389) ${ }^{21}$.

Tous échouent:

iaciuntque sine ordine tela

turba nocet iactis et quos petit impedit ictus (v. 389-390) ${ }^{22}$.

Cette fois le texte ne contient aucune indication impliquant la déesse dans ces insuccès, ce qui est pire pour les prétentions masculines affichées dans les mutuelles exhortations évoquées plus haut! Là aussi, Ovide choisit de s'arrêter sur un moment significatif, amorcé par une prise de parole qui incrimine Diane dans l'insuccès de ces tentatives désordonnées. L'un des chasseurs, Ancée, affirme à la fois la nécessité de rétablir la supériorité masculine, mise à mal par la flèche d'Atalante, et annonce sa propre victoire sur la déesse qui a jusqu'ici protégé le sanglier:

"Discite femineis quid tela uirilia praestent, o iuuenes, operique meo concedite» dixit.

«Ipsa suis licet hunc Latonia protegat armis,

20. "c'est un honneur mérité, s'écrie-t-il, que ta valeur recevra!"

2I. «les hommes ont rougi, s'exhortent et s'appliquent, en y ajoutant des clameurs, de tout leur cœur [...]».

22. «ils jettent leurs traits sans ordre; leur troupe en proie à la confusion nuit à ceux qui ont été lancés et gêne les coups recherchés». 
inuita tamen hunc perimet mea dextra Diana» (v. 392-395) ${ }^{23}$.

La déclaration attribuée à Ancée, un Arcadien, qui veut l'emporter à la fois sur Atalante et sur Diane, est l'un des passages les plus significatifs de l'interprétation d'Ovide, qui raconte la chasse de Calydon comme une guerre des sexes où la position supérieure - déesse oblige - est tenue par les femmes. Ce que confirme une mort immédiate, qui atteint l'insolent au lieu même de sa virilité: le sanglier dirige ses deux défenses summa [...] ad inguina (v. 400) ${ }^{24}$. Le comportement d'Ancée est, en outre, jugé négativement et par l'auteur, qui le qualifie de furens (v. 391) et de tumidus (v. 396), et par un autre chasseur dont la valeur est incontestée, Thésée, qui conseille à son ami le plus cher, Pirithoüs, de combattre, en tant que fortis, de loin: ... licet eminus esselfortibus (v. 406-407) ${ }^{25}$, une formule qui est quasiment une contradiction dans les termes. L'échec des deux coups suivants, dus précisément à des fortes qui combattent de loin, démontre l'inutilité de cette nouvelle tactique, excepté qu'elle préserve effectivement la vie de ceux qui l'adoptent. Le javelot de Thésée est arrêté par une branche, celui de Jason, dérivé par un hasard, dans lequel il faut sans doute voir la main de Diane, vers les flancs d'un de ses compagnons.

Dans un tel contexte, la réussite d'un des traits de Méléagre ne peut que prêter à suspicion: si elle l'a laissé faire, quelle est l'intention de Diane? Contrairement à celui d'Atalante, le succès de Méléagre réjouit tous ses compagnons. C'est alors que le vainqueur prend pour la troisième fois la parole et annonce la mise à exécution de la phrase qui avait déclenché l'agitation brouillonne et téméraire des autres chasseurs, en invitant Atalante à recevoir la dépouille sur laquelle il s'est acquis des droits et à partager sa gloire:

"Sume mei spolium, Nonacria, iuris»

dixit «et in partem ueniat mea gloria tecum» (v. 426-427) ${ }^{26}$.

23. "Apprenez combien des traits lancés par les hommes l'emportent sur ceux des femmes, ô jeunes gens, et inclinez-vous devant mon ouvrage!, dit-il. La fille de Latone en personne peut le protéger des armes, il périra par ma main en dépit de Diane.»

24. "vers le haut de l'aine».

25. "il est permis aux hommes courageux de se tenir à distance».

26. "Prends la dépouille puisque je me suis acquis le droit d'en disposer, Nonacrienne, et qu'ainsi je partage ma gloire avec toi.» 
Si la jeune fille est contente et du présent et de qui le lui offre (un détail qui suggère discrètement que le vainqueur lui plaît), les autres chasseurs sont jaloux. Ovide isole une parole dans le murmure qu'a suscité la décision de Méléagre, qu'il attribue aux fils de Thestius, autrement dit, aux oncles maternels de Méléagre:
«Pone age nec titulos intercipe, femina, nostros»
Thestiades clamant, «nec te fiducia formae
decipiat, ne sit longe tibi captus amore
auctor» ... (v. 433-436) ${ }^{27}$.

Peu importe qu'il soit dans la réalité impossible que ces mots aient été prononcés par les deux frères à l'unisson. Ce qui compte, c'est qu'ils soient, tous les deux, les porte-parole d'une contestation unanime de l'initiative de Méléagre. Cette contestation se fonde - dans le droit fil du récit - sur une opposition femme/homme fondée sur la conviction d'une inégalité des sexes qui n’a même pas à être prouvée, ce qui évidemment invalide le motif, mis en avant par Méléagre, d'une valeur par laquelle la jeune fille se serait distinguée des autres chasseurs. La brutale allusion à une confiance, placée non dans cette valeur, mais dans sa beauté et à la croyance qu'elle peut compter sur l'aide de Méléagre parce qu'à cause de cette beauté, il est tombé amoureux d'elle, est une faute provoquée par la colère, comme le suggèrent les bras tendus (tendentes bracchia, v. 432) des fils de Thestius et l'éclat de leur voix (ingenti [...] uoce, v. 432). Bien que ces mots ne lui soient pas adressés, Méléagre se trouve impliqué: accusé de se retrouver "captif» de ses sentiments, dans une situation de soumission, alors que le jeune homme avait présenté son présent comme une façon de faire accéder Atalante au degré de gloire (supérieur) qu'il avait acquis en tuant l'animal qu'elle avait seulement blessé.

Les fils de Thestius joignent le geste à la parole et, signale Ovide, en enlevant à la jeune fille son munus, enlèvent au vainqueur son ius muneris (v. 436). Saisi de colère à son tour (tumida frendens ... ira, v. 437) ${ }^{28}$, Méléagre s'écrie:

27. «Pose à terre, allons et ne t'empare pas, femme, de nos titres de gloire, s'écrient les fils de Thestius, que la confiance que tu places dans ta beauté ne t'abuse pas; il se pourrait que tu n'aies plus longtemps de garant pris par l'amour."

28. "gonflé de colère, grinçant des dents». 
«Discite, raptores alieni [...] honoris, facta minis quantum distent» (v. 438-439) ${ }^{29}$.

On appréciera la façon dont le poète met en évidence la redistribution, dans cette séquence du mythe, des ingrédients de départ de toute l'histoire: un honor non rendu, de l'ira et le désir de se venger. Il est alors tout à fait en accord avec la perspective développée jusque-là par Ovide que Méléagre tue ses oncles d'une façon qui ne confirme pas sa uirtus. Le jeune homme plonge son épée dans la poitrine du plus proche, qui ne s'y attendait pas (pectora Plexippi nil tale timentia, v. 440) et ne laisse pas à l'autre le temps de se ressaisir (v. 441-444).

Le récit change ensuite de lieu et de personnage. Ovide évoque la mère de Méléagre, sœur de Plexippe et Toxée, à un moment dramatique: elle est en train de porter aux temples des présents nato uictore (v. 445) ${ }^{30}$, quand elle voit ramener les corps inanimés (exstinctos, v. 446) de ses frères. Le renversement sur lequel est construit ce début inaugure ce qui sera le modèle de composition de tout l'épisode: Althaea ne va plus cesser d'osciller d'un sentiment à un autre. Avec l'adjectif exstinctos est introduit le motif du feu qui, à l'inverse des Fastes, n'est pas présent dans l'épisode initial des Métamorphoses, où il est question seulement d'autels laissés sans encens ${ }^{31}$. C'est à ce moment-là de l'histoire qu'Ovide raconte à son lecteur un épisode antérieur: l'apparition des Parques dans la chambre d'Althée, à peine accouchée. Là encore le choix narratif est celui de la scène, ponctuée par une parole significative. Les déesses mettent une bûche dans le foyer, tournent un fil autour de leur pouce en disant :

«Tempora [...] eadem lignoque tibique o modo nate, damus» (v. 454-455) ${ }^{32}$.

Puis elles se retirent. Pas un regard, ni un mot à la mère, mais comme elles sont venues dans sa chambre pour ce geste et cette parole, les déesses ont choisi de la prendre à témoin d'une prophétie, restée sinon

29. "Apprenez, vous qui arrachez une marque d'honneur qui est à autrui, quelle distance il y a des menaces aux actes."

30. "à cause de la victoire de son fils».

3I. [...] solas sine ture relictas / [...] aras (v. 277-278).

32. "Nous donnons la même durée de temps à ce bois et à toi, ô nouveau-né!» 
secrète. Althée arrache le tison du feu, un détail propre au récit ovidien, et le garde caché penetralibus ... imis (v. 458) ${ }^{33}$.

Quand elle apprend que son fils a tué ses frères, son premier geste est de tirer la bûche de sa cachette et d'allumer un feu. Ovide évoque d'abord de l'extérieur la lutte qui se déroule dans l'âme d'Althée entre la mère et la sœur (pugnat materque sororque, v. 463). Il décrit des manifestations physiques successives, qu'il décrypte comme autant de signes d'émotions violentes, selon un code que le lecteur est censé partager et qui est rappelé par un mot ou une expression dénotant un sentiment: de la pâleur (pallebant, v. 465) provoquée par un effroi (metu, v. 465) devant le crime; une rougeur des yeux (ruborem, v. 466) due à une brûlante colère (feruens [...] ira, v. 466); des expressions de cruauté (similis crudele minanti, v. 467) ou suscitées par la pitié (quem misereri credere posses, v. 468); l'absence (ferus ... ardor, v. 469) ou le retour des larmes (lacrimae, v. 470) en relation avec les mêmes sentiments. La rapidité du passage de l'un à l'autre, signalée par les adverbes saepe [...] saepe (v. 465466) et modo [...] modo (v. 467-468) est un autre signe de la force de ces émotions, dont Ovide compare l'instabilité (dubiis affectibus, v. 473) - à celle d'une carène ballottée par des vents contraires (v. 470-471). La répétition du mot ira (v. 466 et 474) confirme qu'Althaéa est un autre substitut de la déesse.

Cette lutte entre deux émotions contraires s'oriente vers une victoire de la «sœur" sur la «mère» (incipit esse tamen melior germana parente, v. 475) ${ }^{34}$. C'est une formulation qui amorce une nouvelle vision du conflit intérieur dont Althaéa est l'objet. La même valeur, la pietas, est en jeu des deux côtés. Le paradoxe qu'implique cette situation est exprimé par une brillante sentence: impietate pia est (v. 477). C'est aussi une façon de montrer que la vengeance de Diane n'a pas pour seul canal la diffusion de sa colère, qui vient de toucher successivement les fils de Thestius, Méléagre et sa mère. Loin de compenser l'absence de pietas d'CEnée, c'est précisément celle de sa femme qui permet à la déesse de se venger, sa piété la plaçant alors face à deux devoirs totalement incompatibles.

Au moment de passer à l'acte, devant des feux qu'elle évoque comme ceux d'un autel funéraire (ante sepulcrales [...] aras, v. 480), Althée

33. "au fin fond de sa maison». Ch. Segal propose de voir dans ce lieu secret une métaphore de l'utérus maternel ("Ovid's Meleager and the Greeks", p. 326).

34. "cependant la sœur commence à l'emporter sur la mère». 
prononce ces mots: Rogus iste cremet mea uiscera! (v. 478) ${ }^{35}$, qui évoquent Méléagre comme une partie d'elle-même. S'ensuit un monologue à la fois conçu sur le modèle tragique (ce qui permet à Ovide de faire entrer le lecteur dans la pensée du personnage) et nourri par la pratique des controverses (examinant donc le pour et le contre). Tout au long de ce monologue, Althée passe d'une décision à une autre, en proie à un trouble qui ne cesse d'augmenter. Elle condamne son fils quand elle envisage la situation en se plaçant du point de vue des pères, et l'épargne quand elle se souvient qu'elle est sa mère. Elle juge en effet intolérable qu'Enée se réjouisse et que Thestius soit dans le deuil:

an felix CEneus nato uictore ${ }^{36}$ fruetur,

Thestius orbus erit? melius lugebitis ambo (v. 486-487) ${ }^{37}$.

Ce qu'elle ne supporte pas - elle le dit peu après - c'est que son fils puisse porter les espérances de son père en lui succédant à la tête de la cité, quand ses propres frères ne sont plus que cendres:

Ergo impune feret uiuusque et uictor et ipso successu tumidus regnum Calydonis habebit, uos cinis exiguus gelidaeque iacebitis umbrae? Haud equidem patiar; pereat sceleratus et ille spemque patris regnumque trahat patriaeque ruinam! (v. 494-497) ${ }^{38}$

Il est frappant que la maternité ne soit pas évoquée en termes de sentiment, mais de mens ("dispositions d'esprit»), avec l'expression mens materna au vers 499, et de iura ("devoirs», le mot étant, qui est plus, modalisé par un génitif qui inclut le père: pia iura parentum au vers

35. "Que ce bûcher brûle le fruit de ma chair!» Ch. Segal cite, entre autres, pour exemple de l'emploi de uiscera pour désigner les enfants, le vers 50 des Remedia amoris à propos de Médée: nec dolor armasset contra sua uiscera matrem ("Ovid's Meleager and the Greeks, p. 325, n. 65).

36. Nato uictore est l'expression dont Ovide s'est servi au début de l'épisode quand il évoque les premiers dons qu'Althée avait prévu d'apporter sur les autels des dieux.

37. "est-ce qu'Enée se réjouira de la victoire de son fils alors que Thestius sera privé d'enfants? Mieux vaut que vous soyez tous les deux en deuil.»

38. "Il sera donc impuni, vivant et vainqueur; gonflé d'orgueil par son succès même il aura le royaume de Calydon, tandis que vous, vous ne serez qu'un petit tas de cendres, des ombres glacées gisantes. Non, je ne le souffrirai pas! Qu'il périsse puisqu'il a commis un crime et qu'il entraîne avec lui les espérances de son père, son royaume, la ruine de sa patrie!» 
499 aussi). Ovide choisit de reprendre d'Euripide ${ }^{39}$ le motif des épreuves de la grossesse ${ }^{40}$ et laisse de côté, même s'il lui aurait fallu l'adapter puisque Méléagre n'est pas aux côtés de sa mère lors de ce monologue, le passage où Médée touche le corps de ses enfants et est alors envahie par une émotion qu'elle ne maîtrise pas ${ }^{41}$. Il met l'accent sur le fait que Méléagre doit deux fois la vie à sa mère: uixisti munere nostro (v. 502), en jouant sur deux sens du mot munus (la phrase pouvant signifier: «tu as vécu grâce à la faveur que je t'ai faite» ou "avec une obligation à mon égard »). En tant que mère, Althée a le droit de disposer d'une vie qu'elle a - doublement - donnée: bisque datam, primum partu, mox stipite rapto, $/$ redde animam (v. 504-505) ${ }^{42}$. Il est tentant de voir, dans le choix propre à Ovide - de placer le retrait du tison du feu immédiatement après l'accouchement, une façon d'en faire le symbole des premiers soins qui maintiennent un enfant en vie mais aussi de tous ceux auxquels il doit ensuite d'être devenu adulte, autrement dit, le symbole de ce que tout enfant doit à celle qui l'a porté, mis au monde, nourri et élevé. La requête d'Althée souligne ce qui en résulte en contrepartie: le droit pour toute mère de demander des comptes. Evidemment, l'histoire de Méléagre est un exemple extrême, cette reddition de comptes entraînant la mort du héros merito tuo (v. 503) à cause de ce qu'il a accompli... et qui la mérite!

Parce qu'elle vient de sa mère, cette mort, comme on l'a souligné, n'a rien à voir avec celle qu'un homme peut rencontrer au combat. C'est une flamme absente et dont il ne sait rien (inscius, v. 515) qui brûle les entrailles de Méléagre (uiscera, v. 516). Il a beau surmonter d'abord ses douleurs par son courage (v. 517-518), il finit sa vie dans les plaintes, appelant son père, ses frères, ses sœurs, sa compagne et, ironie du sort

39. "A quoi me sert, ô mes petits, de vous avoir nourris, d'avoir peiné, d'avoir souffert, de m'être usée, de m'être déchirée dans les douleurs en vous mettant au monde?» (1029 sqq., trad. Marie Delcourt-Curvers).

40. Ubi sunt [...] quos sustinui bis mensum quinque labores? (v. 499-500); "Où sont [...] les peines que j'ai supportées durant deux fois cinq mois?»

4I. "Mes enfants, donnez votre main droite, que votre mère l'embrasse, ô main chérie, bouche chérie, ô beauté, ô noblesse des traits de mes enfants [...]. Contact délicieux, tendre peau, douce respiration de mes enfants [...]. Rentrez, rentrez, je ne puis plus soutenir votre vue.» (v. 1069-1073 et 1074-1076).

42. "rends une vie que je t'ai deux fois donnée, une première fois en accouchant, et juste après en enlevant le tison du feu». 
qu'Ovide n'a pas manqué d'imaginer et qui souligne la faiblesse du héros mourant, peut-être sa mère. C'est par un coup plus viril que la mort atteint Althée aussi dans ses uiscera ${ }^{43}$ : elle s'enfonce, elle-même, une épée dans le ventre (acto per uiscera ferro, v. 532).

Le registre plaintif qui succède au monologue tragique d'Althée se poursuit avec l'évocation des manifestations de deuil qui éclatent dans tout Calydon, achevant la vengeance de la déesse. Celles qui dominent sont attribuées à des femmes, ses sœurs :

Non mihi si centum deus ora sonantia linguis

ingeniumque capax totumque Helicona dedisset, tristia persequerer miserarum dicta sororum (v. 533-535) ${ }^{44}$.

Comme on l'a noté, Ovide reprend un motif qui remonte à Homère ${ }^{45}$ en passant par Ennius ${ }^{46}$ et Virgile ${ }^{47}$, ce qui revient à conjuguer à un souci de variation (après la prise de parole d'Althée, le choix narratif ici est celui de l'ellipse) un renvoi à l'épopée qui donne à cette ellipse la force de la tradition épique. Si Ovide ne détaille donc pas les paroles des sœurs de Méléagre, il évoque en revanche tous leurs gestes, qu’on pourrait qualifier de «maternels» à l'égard du corps de leur frère:

corpus refouentque fouentque, oscula dant ipsi, posito dant oscula lecto (v. 537-538) ${ }^{48}$.

Elles en viennent dans leur douleur à un paroxysme qui leur fait prodiguer leur tendresse aux cendres du bûcher (elles les pressent sur leur poitrine), puis au tombeau lui-même sur lequel elles s'allongent et restent

43. Ch. Segal note qu'Ovide semble être le seul écrivain à attribuer cette mort à Althée ("Ovid's Meleager and the Greeks», p. 328).

44. "Non, si un dieu m'avait donné cent bouches aux langues retentissantes, un vaste génie et tout l'Hélicon, je ne pourrais pas répéter les tristes plaintes de ses malheureuses sœurs.»

45. Homère, Iliade, 2.488.

46. An., 561-562.

47. Virgile, Géorgiques, 2.42-44; Enéide, 6.625-626. On trouve aussi ce motif dans un passage des Géorgiques (2.42-44) où Virgile évoque la tâche qui l'attend en recourant à une métaphore qui renvoie aussi à l'épopée: celle du navire volant sur la mer, toutes voiles déployées.

48. "elles réchauffent de leurs caresses encore et encore le corps, lui donnent des baisers, en donnent au lit une fois qu'il y a été posé.» 
étendues, répandant leurs larmes sur la pierre où est inscrit le nom de leur frère (v. 539-541). Comme l'a souligné Nicole Loraux ${ }^{49}$, le deuil fait, en Grèce, partie des conduites qui risquent d'entamer l'ordre de la cité: perçu comme d'essence féminine, il est réglé par diverses législations qui tendent à le limiter dans l'espace et dans le temps. C'est l'inverse qui se passe ici, avec le déferlement de manifestations féminines qui ne connaissent absolument aucune limitation. Et c'est ce signe patent du bouleversement complet de l'organisation mâle de la cité, qui assouvit enfin la colère de Diane, Parthaoniae [...] Latonia clade (v. 542).

Dans sa cinquième épinicie, Bacchylide fait se rencontrer aux Enfers Méléagre et Héraclès. La destinée du premier inspire au second une réflexion amère sur l'existence des mortels, pour qui «le mieux est de n'être pas né " ${ }^{50}$. C'est seulement implicitement que la destinée de Méléagre, achevée par la main d'une femme, préfigure celle d'Héraclès. Ovide reprend cette perspective générale en se servant de l'élément qui n'est pas pris en considération par Bacchylide: les rapports de Méléagre aux femmes. L'intrusion d'Atalante, dans l'univers traditionnellement viril des chasseurs de bêtes sauvages monstrueuses, met à mal la confortation de l'identité masculine, opérée dans et par ce genre d'aventures, excepté pour Méléagre. Mais la suite du récit démontre que, de sa naissance à ses funérailles, la vie du jeune homme tout entière aura été régie par des rapports aux femmes, qui sont indépendants de sa volonté et dont il n'a même pas conscience.

Le tison magique auquel sa vie est attachée est l'image de cette domination secrète: pris en charge sans que nul ne le sache par la mère de Méléagre, il est l'emblème de ces liens invisibles et tout-puissants que chaque homme a, de sa naissance à sa mort, avec les femmes de sa famille ${ }^{51}$. Comme le montre le geste des déesses qui délèguent leur droit

49. N. Loraux, Les mères en deuil, p. 35.

50. Bacchylide, Epinicies, 5.160.

5I. Dans l'histoire de Méléagre, la place occupée par Atalante est celle de l'épouse, si l'on considère que les vers 326-327 expriment le souhait du héros et si c'est bien elle que désigne au vers 521 l'expression sociamque tori. Quoi qu'il en soit, l'ambiguïté sur son statut est un choix de l'auteur, qui joue ainsi avec cette possibilité. Les variations sur ce thème du pouvoir caché des femmes sont nombreuses. Sur l'importance des figures féminines du destin, humaines ou divines, qui président à la naissance dans le monde grec et romain, voir dans ce volume les articles de V. Pirenne-Delforge et G. Pironti et de V. Dasen. 
d'impartir une durée à l'existence humaine, immédiatement après la naissance de Méléagre, à celle qui vient de lui donner la vie, les Parques et Althée sont en fait interchangeables. Quant aux sœurs, elles sont des doublets de la mère dont elles prennent en charge les gestes de soins et de tendresse. Dans les Métamorphoses, Althée relaie Atalante comme instrument involontaire de la vengeance de Diane. Dans ses Dialoghi con Leucò, C. Pavese ${ }^{52}$ ira plus loin qu'Ovide, en voyant dans la jeune chasseresse une autre Althée, capable, elle aussi, de regarder un jour brûler le même genre de feu. A l'affirmation de Méléagre, s’indignant que les yeux de mère aient pu fixer le foyer où se consumait le tison - «è inaccettabile che fissino il fuoco vedendo il tisone [...] ma non credo che anche lei - la giovane - sapesse quegli occhi» - , son interlocuteur divin, Hermès, qui le conduit aux Enfers, rétorque: "Non li sapeva. Era quegli occhi.»

Jacqueline FABRE-SERRIS

Université de Lille 3

52. C. Pavese, Dialoghi con Leucò, p. 54-55. 


\section{BIBLIOGRAPHIE}

\section{Sources}

Bacchylide, Epinicies, texte établi par J. Irigoin, traduit par J. Duchemin, L. Bardollet, Paris, Les Belles Lettres, 1993.

Euripide, Tragédies, Médée, texte traduit et annoté par M. Delcourt-

Curvers, Paris, Le livre de poche, 1969.

Homère, Iliade, texte établi par P. Mazon, Paris, Les Belles Lettres, 1972.

Ovide, Metamorphoses, Book VIII, ed. Adrian S. Hollis, Oxford, Clarendon Press, 1970.

—, Les Métamorphoses, texte établi par G. Lafaye, Paris, Les Belles Lettres, 1994.

Virgile, Enéide, texte établi par J. Perret, Paris, Les Belles Lettres, 1977. —, Géorgiques, texte établi par E. de Saint-Denis, Paris, Les Belles lettres 1974.

Travaux

Conte, Gian Biagio, Profilo storico della letteratura latina, Firenze, Le Monnier, 2004.

Grossard, Peter, Die Erzählung von Meleagros. Zur literarischen Entwicklung der Kalydonischen Kultlegende, Leiden, Brill, 2001.

Loraux, Nicole, Les mères en deuil, Paris, Seuil, 1990.

Pavese, Cesare, Dialoghi con Leucò, Milano, Einaudi, 1979 [1947].

SEgAL, Charles, "Ovid's Meleager and the Greeks: trials of gender and genre", Harvard Studies in Classical Philology, 99 (1999), p. 301-340. 
